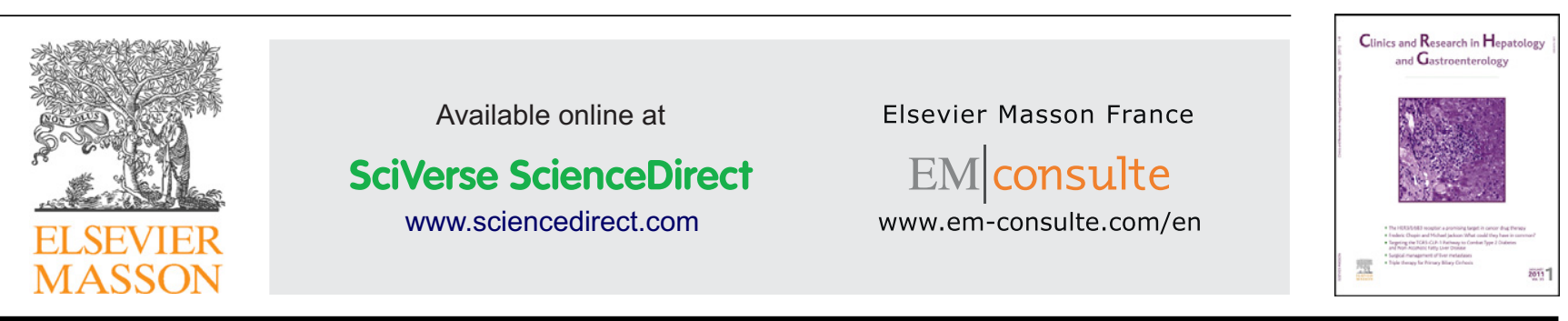

ORIGINAL ARTICLE

\title{
Short-term multidisciplinary non-pharmacological intervention is effective in reducing liver fat content assessed non-invasively in patients with nonalcoholic fatty liver disease (NAFLD)
}

\section{Federica Scaglioni ${ }^{a}$, Mariano Marino a , Stefania Ciccia ${ }^{a}$, Alessia Procaccini ${ }^{a}$, M. Busacchi ${ }^{a}$, Paola Loria ${ }^{b}$, Amedeo Lonardo $^{b, *}$, Marcella Malavolti $^{c}$, Nino Carlo Battistini ${ }^{c}$, Massimo Pellegrini ${ }^{c}$, Francesca Carubbi ${ }^{c}$, Stefano Bellentani ${ }^{a, * *}$}

\footnotetext{
a Liver Centre, "Ramazzini"' Hospital Carpi, Azienda USL Modena, Italy

b Department of Metabolic Medicine, University of Modena and Reggio Emilia, Modena, Italy

c Department of Public Health Sciences, University of Modena and Reggio Emilia, Modena, Italy
}

\begin{abstract}
Summary
Background: Non-alcoholic fatty liver disease (NAFLD) ranges from simple steatosis to steatohepatitis, and cirrhosis in patients with alcohol intake less than $20 \mathrm{~g} / \mathrm{day}$, and is usually associated with insulin resistance (IR).

Aim: Given that no drugs are specifically approved for NAFLD, we tested the efficacy of a nonpharmacological multidisciplinary intervention based on a personalized diet, physical activity and behavior therapy.

Methods: In this open non-randomized study, personalized diet, physical exercise and behaviour therapy for 3 months were prescribed in 12 consecutive patients with NAFLD. Lifestyle, including total caloric intake, physical activity and resting energy expenditure was monitored by a SenseWear Armband. Insulin Resistance (IR) was measured by HOMA and oral glucose insulin sensitivity tests (OGIS); fat liver content was estimated by two different semi-quantitative scores and by the Doppler Power Index (DPI).

Results: Data show that the multidisciplinary intervention produced a significant reduction of total caloric intake, a $8 \%$ reduction in body weight, a modest increase in daily physical activity,
\end{abstract}

\footnotetext{
* Corresponding author. Internal Medicine, University of Modena and Reggio Emilia, Modena, Italy.

** Corresponding author. Liver Centre, "Ramazzini" ' Hospital, Gastroenterology, P. le Donatori di Sangue n 3, 41012 Carpi, Modena, Italy. Tel.: +39 059 659250/659372; fax: +39 059851762.

E-mail addresses: a.lonardo@libero.it (A. Lonardo), bellentanistefano@gmail.com, s.bellentani@ausl.mo.it (S. Bellentani).
} 
a significant $(P<0.001)$ reduction of aminotransferases and a decrease of total hepatic fat content.

Conclusions: A 3-month multidisciplinary intervention inducing at least $8 \%$ of weight loss, improves liver tests and decreases liver fat content.

(c) 2012 Published by Elsevier Masson SAS.

\section{Background}

Non-alcoholic fatty liver disease (NAFLD) is defined by intrahepatocyte accumulation of lipids, in patients who have no or only modest (<20 grams of alcohol per day) [1,2] alcohol intake, in the absence of competing etiology of liver disease. Rather than being a mere "hepatic manifestation" of the Metabolic Syndrome of insulin resistance (IR) actually NAFLD is more likely to be an essential prerequisite for the development of such a syndrome which rarely occurs, if ever, in humans, in the absence of NAFLD [3]. Hence, NAFLD is a novel risk factor for cardiovascular diseases (CVD) [4]. Histologically, NAFLD describes a large spectrum of diverse conditions, ranging from simple steatosis, to non-alcoholic steatohepatitis (NASH), fibrosis, cirrhosis, and hepatocellular carcinoma (HCC) [5-9].

The prevalence of NAFLD is progressively increasing, paralleling the surge of "diabesity" and related metabolic diseases. The clinical presentation of NAFLD is deceptively silent. Diagnosis is based on a modest alteration of aminotrasferases and/or the occasional finding of "bright liver"' at ultrasound liver scanning. Once the diagnosis is made, however, no specific drugs are approved for use in NAFLD [10-12]. Non-pharmacological intervention based on personalized diet, physical activity and behavior therapy aimed at changing lifestyle is the only therapy proven to be effective $[13,14]$. In their recent systematic review, Thoma and Trenell [15] identified 23 studies that consistently showed reduction in liver fat content, aminotransferase levels, and improved glucose metabolism and insulin resistance. However, only seven out of the 23 studies aimed to obtain a change in lifestyle in order to decrease caloric intake and promote increased physical activity over a 3 to 12 month period. Furthermore, in these studies, the hepatic fat content was measured either invasively with liver biopsy or non-invasively with Proton Magnetic Resonance Spectroscopy ( $\left.{ }^{1} \mathrm{H}-\mathrm{MRS}\right)$, and Computed Tomography (CT), two techniques which are expensive, not universally available, or expose the patient to X-rays [15]. at:

On these grounds, this pilot open label study was aimed

- establishing a personalized short-term (3 months) nonpharmacological intervention based on diet, physical activity associated with psychological counseling for the treatment of NAFLD;

- assessing the effects of this multi-step and multidisciplinary intervention program on liver fat.

\section{Methods}

The study was approved by the local Ethical Committee. Twelve consecutive patients seen at our Liver Center in Campogalliano (Modena, Italy) from May 2009 until October 2009, with different degrees of hepatic steatosis on ultrasound were enrolled.

All the 12 patients enrolled were asymptomatic and had been referred to our outpatient service by their general practitioners. All of our patients had had previous experience with self-prescribed diet without success, but none of them had followed lifestyle change programs before. In agreement with recent guidelines [14,16] the diagnosis of NAFLD was reached by ultrasound, after exclusion of competing etiologies of liver disease. After a baseline assessment, patients were requested to sign a written informed consent, and to follow a 3-month multidisciplinary non-pharmacological treatment based on personalized diet, physical activity, and behavior therapy. A short-term period of intervention was purposefully selected on the grounds that long-term multidisciplinary intervention is usually curtailed by much lower compliance rates.

The diet was indicated by our dietician after a food intake analysis based on a 7-day food diary.

The daily physical activity (PA) was monitored at baseline, and after 1 and 3 months, through both a 7-day PA diary, and a specific tool named Armband or SWA (SenseWear ${ }^{T M}$ Armband, BodyMedia, Roche Diagnostics, Indianapolis, IN, USA) [17]. This device works as a "metabolic recorder" and as a step-meter. The Armband was worn on the right arm over the triceps muscle at the midpoint between the acromion and olecranon processes, and kept for 5 to $10 \mathrm{~min}$ before data collection in order to allow acclimation to skin temperature. The SWA data were analysed using dedicated most updated software version (InnerView Research Software, version 6.0 BodyMedia, Inc., Pittsburgh, PA) [18]. SWA also provided a number of measured parameters (biaxial acceleration, heat flux, galvanic skin response, skin temperature, near body temperature) which were elaborated as a function of the demographic and anthropometric characteristics (gender, age, height, weight) of the individual patient by proprietary algorithms estimating energy expenditure (total energy expenditure, energy expenditure in physical activity and resting or basal energy expenditure).

Motivation to lifestyle changes, psychological counseling and support were provided to each patient by a specifically trained and expert cognitive-behavioral psychologist, at baseline, and after 1 and 3 months. The behavior component of lifestyle modification for weight loss includes all the 
Table 1 Composition of the diet at baseline and after 3 months of multidisciplinary intervention as calculated with the 7-day food diary. Last column shows statistical significance (two tails) of the data at 3 months vs. baseline as calculated by $T$ test for paired data.

\begin{tabular}{lccc}
\hline Daily energy intake & Baseline & 3 months & $P$ \\
\hline Total (Kcal) & $2752 \pm 403$ & $2243 \pm 288$ & 0.001 \\
Fat (Kcal) & $936 \pm 243$ & $663 \pm 162$ & 0.001 \\
Carboydrates complex (Kcal) & $1353 \pm 162$ & $1163 \pm 143$ & 0.001 \\
Proteins (Kcal) & $463 \pm 91$ & $416 \pm 87$ & 0.145 \\
Dietary fibers (g) & $22.8 \pm 5.4$ & $21.2 \pm 4.2$ & 0.366 \\
Saturated fatty acids (Kcal) & $189 \pm 126$ & $38 \pm 31$ & 0.031 \\
Monounsaturated fatty acids (Kcal) & $497 \pm 109$ & $380 \pm 53$ & 0.001 \\
Poliunsaturated fatty acids (Kcal) & $136 \pm 31$ & $103 \pm 25$ & 0.001 \\
\hline
\end{tabular}

procedures found to be effective to reach the patients' motivation to lifestyle changes [13,19-21]. Particularly, adherence to dietary and exercise guidelines should be favored by clear, easy-to manage recommendations, tailored on patients' preferences.

All patients underwent consultations with the hepatologist, psychologist and dietician at baseline and after 1 and 3 months. Blood tests, ultrasound scanning, anthropometric and Armband evaluation were also performed at time 0 and 3 months. The basal assessment of fibrosis was determined by Fibroscan elastography, a non-invasive predictor of liver fibrosis [22].

IR was assessed by means of HOMA and OGIS tests. [23-26]. All anthropometric measurements were performed by the same operator according to the Anthropometric Standardization Reference Manual [27]. Weight (Wt) was measured to the nearest $100 \mathrm{~g}$ and height $(\mathrm{Ht})$ to the nearest $0.1 \mathrm{~cm}$ using an electronic scale with built-in stadiometer (Tanita, Tokyo, Japan). Body Mass Index (BMI) was calculated as $\mathrm{Wt}(\mathrm{Kg}) / \mathrm{Ht}\left(\mathrm{m}^{2}\right)$. Circumferences (arm, waist, hip) were measured to the nearest millimeter using a plastic tape measure. All skin-folds and waist circumference measurements were taken three times and the values were averaged. HOMA and OGIS were performed with the patient fasting and at rest on an outpatient basis after an oral 75-g glucose load. Serial blood samples were collected at baseline, and at 30, 60, 90, 120 and 180 minutes following glucose load. At baseline blood was also sampled to test liver enzymes, renal function, and lipid profile. Fatty Liver Index was calculated according to the usual formula [28,29], reported underneath: $\mathrm{FLI}=\left(\mathrm{e} \quad 0.953^{*} \operatorname{loge}(\right.$ triglycerides $)+0.139^{*} \mathrm{BMI}+0.718^{*} \operatorname{loge}(\mathrm{GGT})+0.053^{*}$ waist

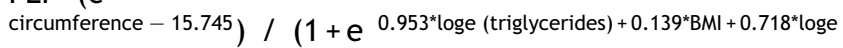
$(\mathrm{GGT})+0.053^{*}$ waist circumference -15.745$) * 100$.

The presence of fatty liver was assessed by ultrasound (US) performed by the same operator to avoid intra- or inter-rater disagreement. The quantification of liver fat was performed according to Hamaguchi et al. [30], and a novel score recently developed and validated by our group, named US-FLI [31]. US-FLI ranges 2 to 8 and a score $<4$ rules out NASH with high sensitivity and specificity [31].

To quantify hepatic steatosis with Doppler-US, we used the Doppler Power Index (DPI), developed by Kakkos et al. [32], and estimated the elasticity of blood vessels, particularly the elasticity of portal vein and hepatic artery.

\section{Statistical analysis}

The distribution of each continuous variable was assessed with the non-parametric Kolmogorov-Smirnov procedure [33]. Because the variables were normally distributed, all the continuous nominal variables were reported as mean \pm SD. Correlations were assessed using Spearman's Rho test for non-parametric variables, while for the parametric test Pearson's test was used. To evaluate differences between time 0 and time 3 months, the $T$ test for paired samples was used. Statistical significance was set to a twotailed value of $P<0.05$ for all tests. Statistical analysis was performed using STATA 9.2 (STATA Corp., College Station, $\mathrm{TX})$.

\section{Results}

The mean age of our subjects was 47.25 years ( \pm 13.57 SD), with predominance of male sex $(80 \%)$, a sedentary lifestyle (baseline daily METS =1.5) with a high (saturated) fat, high calorie diet at baseline. These individuals did not have diabetes, dyslipidaemia or other known diseases, but only slightly altered liver tests. Liver stiffness measurement in our patients was less than $6.0 \mathrm{KPa}$, indicating absence of, or stage 1 fibrosis [22].

As shown in Table 1, the average daily intake expressed in Kcal significantly decreased after a 3-month personalized diet from 2752 to $2243(P<0.001,18.5 \%$ of reduction). The highest reduction was observed in fat intake (30\%) and complex carbohydrates (14\%).

A highly significant correlation $(r=0.930, P=0.0001)$ was found between the PA estimated in METS by the SWA with that of the personal 7-day PA diary, both at baseline and after 3 months, suggesting that Armband is a valid device to measure daily PA. However, all our patients already had a basal minimal or null physical activity (METs ranging between 1.2 to 1.8 ) which, after a 3-month multidisciplinary intervention, increased by $+9.5 \%$ [expressed both in number of daily steps $(10,554 \pm 4942$ vs. $11,560 \pm 4212)$, and in METS $(1.50 \pm 0.27$ vs. $1.58 \pm 0.25)$ ] to a statistically nonsignificant extent: Moreover, patients did not engage in at least a moderate-intensity activities, (ranging between 3 to 6 METs). This indicates, as also suggested by the psychological assessment, that motivation in changing PA was lower 
Table 2 Results of laboratory data parameters at baseline and after 3 months of multidisciplinary intervention. Last column shows statistical significance (two tails) of the data at 3 months vs. baseline as calculated by $T$ test for paired data.

\begin{tabular}{lccc}
\hline Variables & Baseline & 3 months & $P$ \\
\hline Cholesterol (mg/dL) & $196 \pm 34$ & $197 \pm 27$ & 0.933 \\
Tryglicerides (mg/dL) & $193 \pm 130$ & $137 \pm 56$ & 0.238 \\
AST (U/L) & $32 \pm 15$ & $26 \pm 9$ & 0.053 \\
ALT (U/L) & $48 \pm 41$ & $35 \pm 32$ & 0.012 \\
GGT (U/L) & $28 \pm 12$ & $26 \pm 9$ & 0.494 \\
Acid (mg/dL) & $6.5 \pm 1.3$ & $6.5 \pm 1.2$ & 0.958 \\
Insulin (ug/dL) & $8.2 \pm 4.1$ & $9.9 \pm 6.1$ & 0.056 \\
Glycemia (mg/dL) & $98.6 \pm 12.4$ & $93.6 \pm 11.7$ & 0.072 \\
HOMA & $1.95 \pm 0.6$ & $2.32 \pm 1.4$ & 0.319 \\
OGIS & $8.39 \pm 0.8$ & $9.91 \pm 1.6$ & 0.166 \\
Fatty Liver Index (FLI) & $61 \pm 17$ & $47 \pm 20$ & 0.027 \\
\hline
\end{tabular}

than that to changing the diet. The results obtained after 3 months, mainly with diet, were mirrored by changes in the anthropometric indexes: BMI, waist and hip circumference (WC, HP) significantly decreased (28.6 vs. 26.5; 97.6 vs. 92.7 ; and 103.2 vs. 100.1 , respectively, $P<0.01$ ), with a reduction of $7.34 \%$ of both weight and $\mathrm{BMI}$, and of $5.0 \%$ of the WC.

As shown in Table 2, the laboratory markers of liver damage and the glucose-lipid profiles improved after 3 months, even if the difference did not always reach the statistical significance. In all 12 patients, also the 180 minute plasma glucose and insulin curves after a standard glucose load, significantly improved $(P<0.01$, data not shown). However, HOMA and OGIS did not reach the threshold of statistically significance $(P=0.319$ and 0.166$)$, probably owing to the small sample analyzed, and the large variability of individual data.

Interestingly, the two ultrasonographic scores used to quantify liver steatosis (Hamaguchi score and USFLI) and the DPI significantly $(P<0.05)$ correlated $(r=0.817 / 0.875 / 0.905$, respectively). All of these three US scores semiquantitatively indicating liver fat decreased significantly $(P<0.001) 3$ months after the beginning of multidisciplinary intervention (Table 3 ).

\section{Discussion}

This pilot study conducted in 12 patients with NAFLD shows that in the short-term, multidisciplinary nonpharmacological intervention [13], aimed at changing unhealthy lifestyles, based essentially on personalized diet and behavior therapy, improves not only BMI and WC, but also plasma lipid profile, liver tests and liver fat content $[29,34,35]$. The regimen also improved two ultrasound scores which are correlated with liver histology. The significant relationship of the Hamaguchi score [30], with the novel US-FLI score recently proposed by our group [31] and the DPI [32], and the significant reduction of all of them after 3 months of non-pharmacological multidisciplinary intervention, indicate that these scores can be used as non-invasive markers of effective therapeutic intervention.

In agreement with previous reports [36,37], a statistically significant decrease in aminotrasferase and lipids levels was seen in our patients, indirectly suggesting that the activity of the ongoing liver inflammatory changes may be blunted by decreasing intra-hepatic liver fat content.

Many controlled and uncontrolled studies have shown that a high caloric or an unbalanced diet, and a reduced PA may play an important role in NAFLD and NASH. However few studies have explored the effect of a multidisciplinary intervention based on tailored diet, PA and behavior therapy, on BMI, WC, PA, energy expenditure, insulin resistance, liver function and fat content. It is known that specific single dietary and lifestyle factors are independently associated with long-term weight gain, with substantial implications in the strategies to prevent obesity [38]. Promrat. [39] demonstrated that in 31 overweight or obese patients with histologically diagnosis of NASH, all the subjects who underwent an intensive program of lifestyle intervention based on a combination of diet, exercise and behavior modification achieved a minimum weight loss of at least $7 \%$ of the initial body weight, and a significant improvement of steatosis, lobular inflammation, ballooning injury and NASH Activity Score (NAS), at liver histology. Physical exercise can reduce liver fat content [40]. However, while most NAFLD patients

Table 3 Ultrasound scores used to quantify fat in the liver at baseline and after 3 months of multidisciplinary intervention. Last column shows statistical significance (two tails) of the data at 3 months vs. baseline as calculated by $T$ test for paired data.

\begin{tabular}{llll}
\hline Variable & Baseline & 3 months & $P$ \\
\hline Hamaguchi score & $5.08 \pm 0.90$ & $3.08 \pm 1.16$ & 0.001 \\
US-FLI & $6.58 \pm 1.16$ & $3.83 \pm 1.40$ & 0.001 \\
Doppler Power Index (DPI) & $0.27 \pm 0.08$ & $0.15 \pm 0.06$ & 0.010 \\
\hline
\end{tabular}


understand the benefit of physical exercise, they lack confidence/motivation to perform them [41]. Our study confirms this conclusion. We obtained a significant improvement of metabolic parameters, of the FLI, and a more significant reduction of the non-invasive ultrasound scores of steatosis (ranging from 39 to $44 \%$ ) with an average reduction of as little as $8 \%$ of the weight alone.

In conclusion, although performed in a small sample of NAFLD patients, our pilot study showed that a 3-month multidisciplinary intervention aimed at changing lifestyle and based on tailored low calorie low fat diet, increased physical activity and behaviour therapy, resulted in a $8 \%$ reduction in body weight and improved liver enzymes, but also the liver fat content measured by semi-quantitative ultrasound scores. Other long-term studies are necessary to investigate whether these lifestyle changes maintained over time will obtain further improvements, and if patients with NAFLD will be sufficiently motivated to maintain such lifestyle changes over the long run.

\section{Disclosure of interest}

The authors declare that they have no conflicts of interest concerning this article.

\section{Acknowledgements}

The research leading to these results has received funding from the European Community's Seventh Framework Programme (FP7/2007-2013) under grant agreement $n^{\circ}$ HEALTH-F2-2009-241762 for the project named FLIP.

\section{References}

[1] Angulo P. Nonalcoholic fatty liver disease. N Engl J Med 2002;346(16):1221-31.

[2] Neuschwander-Tetri BA, Caldwell SH. Nonalcoholic steatohepatitis: summary of an AASLD single topic conference. Hepatology 2003;37(5):1202-19.

[3] Vanni E, Bugianesi E, Kotronen A, De Minicis S, Yki-Järvinen H, Svegliati-Baroni G. From the metabolic syndrome to NAFLD or vice versa? Dig Liver Dis 2010;42:320-30.

[4] Targher G, Day CP, Bonora E. Risk of cardiovascular disease in patients with nonalcoholic fatty liver disease. N Engl J Med 2010;363(14):1341-50.

[5] Falck-Ytter $Y$, et al. Clinical features and natural history of nonalcoholic steatosis syndromes. Semin Liver Dis 2001;21(1):17-26.

[6] Matteoni CA, et al. Nonalcoholic fatty liver disease: a spectrum of clinical and pathological severity. Gastroenterology 1999;116(6):1413-9.

[7] Ong JP, Pitts A, Younossi ZM. Increased overall mortality and liver-related mortality in non-alcoholic fatty liver disease. $J$ Hepatol 2008;49(4):608-12.

[8] Younossi ZM, et al. Changes in the prevalence of the most common causes of chronic liver diseases in the United States from 1988 to 2008. Clin Gastroenterol Hepatol 2011;9(6):524-30, e1; quiz e60.

[9] Younossi ZM, et al. Pathologic criteria for nonalcoholic steatohepatitis: interprotocol agreement and ability to predict liver-related mortality. Hepatology 2011;53(6):1874-82.
[10] Pascale A, Pais R, Ratziu V. An overview of nonalcoholic steatohepatitis: past, present and future directions. J Gastrointestin Liver Dis 2010;19(4):415-23.

[11] Ratziu V, et al. A position statement on NAFLD/NASH based on the EASL 2009 special conference. J Hepatol 2010;53(2):372-84.

[12] Ratziu V, Caldwell S, Neuschwander-Tetri BA. Therapeutic trials in nonalcoholic steatohepatitis: insulin sensitizers and related methodological issues. Hepatology 2010;52(6):2206-15.

[13] Bellentani S, et al. Behavior therapy in nonalcoholic fatty liver disease: the need for a multidisciplinary approach. Hepatology 2008;47(2):746-54.

[14] Loria P, et al. Practice guidelines for the diagnosis and management of nonalcoholic fatty liver disease. A decalogue from the Italian Association for the Study of the Liver (AISF) Expert Committee. Dig Liver Dis 2010;42(4):272-82.

[15] Thoma CDC, Trenell MI. Lifestyle interventions for the treatment of non-alcoholic fatty liver disease in adults: a systematic review. J Hepatol 2012;56:255-66, http://dx.doi.org/10.1016/j.jhep.2011.06.010.

[16] Chalasani N, Younossi Z, Lavine JE, Diehl AM, Brunt EM, Cusi $\mathrm{K}$, et al. The diagnosis and management of non-alcoholic fatty liver disease: practice Guideline by the American Association for the Study of Liver Diseases American College of Gastroenterology, and the American Gastroenterological Association. Hepatology 2012;55(6):2005-23.

[17] Jakicic JM, et al. Evaluation of the sensewear proarmband to assess energy expenditure during exercise. Med Sci Sports Exerc 2004;36(5):897-904.

[18] Malavolti $M$, et al. A new device for measuring resting energy expenditure (REE) in healthy subjects. Nutr Metab Cardiovasc Dis 2007;17(5):338-43.

[19] Wadden TA, Foster GD. Behavioral treatment of obesity. Med Clin North Am 2000;84(2):441-61, vii.

[20] Fabricatore AN. Behavior therapy and cognitive-behavioral therapy of obesity: is there a difference? J Am Diet Assoc 2007;107(1):92-9.

[21] Prochaska JO, DiClemente CC. Stages and processes of selfchange of smoking: toward an integrative model of change. J Consult Clin Psychol 1983;51(3):390-5.

[22] Kumar R, Rastogi A, Sharma MK, Bhatia V, Tyagi P, Sharma P, et al. Liver stiffness measurements in patients with different stages of nonalcoholic fatty liver disease: diagnostic performance and clinicopathological correlation. Dig Dis Sci 2012 [Epub ahead of print] PMID: 22790906.

[23] Levy JC, Matthews DR, Hermans MP. Correct homeostasis model assessment (HOMA) evaluation uses the computer program. Diabetes Care 1998;21(12):2191-2.

[24] Kanauchi $M$, et al. Homeostasis model assessment of insulin resistance, quantitative insulin sensitivity check index, and oral glucose insulin sensitivity index in nonobese, nondiabetic subjects with high-normal blood pressure. J Clin Endocrinol Metab 2003;88(7):3444-6.

[25] Mari A, et al. A model-based method for assessing insulin sensitivity from the oral glucose tolerance test. Diabetes Care 2001;24(3):539-48.

[26] Svegliati-Baroni G, et al. Post-load insulin resistance is an independent predictor of hepatic fibrosis in virus $C$ chronic hepatitis and in non-alcoholic fatty liver disease. Gut 2007;56(9):1296-301.

[27] Lohman TG, Roche AF, Martorell R. Anthropometric Standardization Reference Manual. Human. Champaign IL: Human Kinetics Books; 1988.

[28] Bedogni G, et al. A simple index of lipid overaccumulation is a good marker of liver steatosis. BMC Gastroenterol 2010;10:98.

[29] Bedogni G, et al. The Fatty Liver Index: a simple and accurate predictor of hepatic steatosis in the general population. BMC Gastroenterol 2006;6:33. 
[30] Hamaguchi $M$, et al. The severity of ultrasonographic findings in nonalcoholic fatty liver disease reflects the metabolic syndrome and visceral fat accumulation. Am J Gastroenterol 2007;102(12):2708-15.

[31] Ballestri S, Lonardo A, Romagnoli D, Carulli L, Losi L, Day CP, et al. Ultrasonographic fatty liver indicator, a novel score whch rules out NASH and is correlated with metabolic parameters in NAFLD. Liver Int 2012;32(8):1242-52.

[32] Kakkos SK, et al. Fatty liver in obesity: relation to Doppler perfusion index measurement of the liver. Scand J Gastroenterol 2000;35(9):976-80.

[33] Glantz S. Primer of Biostatistics. Alternative to analysis of variance and $t$ test based on ranks. New York: MacGraw-Hill; 1981, 269-311.

[34] Calori G, et al. Fatty liver index and mortality: the cremona study in the 15th year of follow-up. Hepatology 2011;54:145-52, http://dx.doi.org/10.1002/hep.24356.

[35] Gastaldelli A, et al. Fatty liver is associated with insulin resistance, risk of coronary heart disease, and early atherosclerosis in a large European population. Hepatology 2009;49(5):1537-44.
[36] Marchesini G, et al. Aminotransferase and gammaglutamyltranspeptidase levels in obesity are associated with insulin resistance and the metabolic syndrome. J Endocrinol Invest 2005;28(4):333-9.

[37] Fracanzani AL, et al. Risk of severe liver disease in nonalcoholic fatty liver disease with normal aminotransferase levels: a role for insulin resistance and diabetes. Hepatology 2008;48(3):792-8.

[38] Mozaffarian D, et al. Changes in diet and lifestyle and long-term weight gain in women and men. $\mathrm{N}$ Engl J Med 2011;364(25):2392-404.

[39] Promrat K, et al. Randomized controlled trial testing the effects of weight loss on nonalcoholic steatohepatitis. Hepatology 2010;51(1):121-9.

[40] Johnson NA, et al. Aerobic exercise training reduces hepatic and visceral lipids in obese individuals without weight loss. Hepatology 2009;50(4):1105-12.

[41] Frith J, et al. Potential strategies to improve uptake of exercise interventions in non-alcoholic fatty liver disease. J Hepatol 2010;52(1):112-6. 